\title{
Efficacité d'une prise unique de Praziquantel pour le traitement de la bilharziose urinaire en zones endémiques chez les enfants d'âge scolaire au Mali
}

\author{
Alpha Seydou Yaro, Maitre de Conférences, PhD \\ Michel Emanuel Coulibaly, Master
}

Faculté des Sciences et Techniques (FST), Université des Sciences, des Techniques et des Technologies de Bamako (USTTB), Mali ; International

Centre for Excellence in Research (ICER-Mali) Point G ; Faculté de

Médecine et d'Odontostomatologie (FMOS)

USTTB, Bamako, Mali

Yaya Coulibaly, Maitre-Assistant, PhD

International Centre for Excellence in Research (ICER-Mali) Point G Faculté de Médecine et d'Odontostomatologie (FMOS) ; USTTB, Bamako, Mali

Bernard Sodio, Professeur Titulaire, PhD

Faculté des Sciences et Techniques (FST), Université des Sciences, des

Techniques et des Technologies de Bamako (USTTB), Mali

Sekou F. Traoré, Professeur Titulaire, PhD

International Centre for Excellence in Research (ICER-Mali) Point G Faculté de Médecine et d'Odontostomatologie (FMOS) ; USTTB, Bamako, Mali

Doi:10.19044/esj.2021.v17n17p162

Submitted: 12 January 2021

Accepted: 27 April 2021

Published: 31 May 2021
Copyright 2021 Author(s)

Under Creative Commons BY-NC-ND

4.0 OPEN ACCESS

Cite As:

Yaro A.S., Coulibaly M.E., Coulibaly Y., Sodio B. \& Traoré S.F. (2021). Efficacité d'une prise unique de Praziquantel pour le traitement de la bilharziose urinaire en zones endémiques chez les enfants d'âge scolaire au Mali. European Scientific Journal, ESJ, 17(17), 162. https://doi.org/10.19044/esj.2021.v17n17p162

\section{Résumé}

$\mathrm{Au}$ Mali, la schistosomiase est un problème de santé publique comme dans tous les 42 paysafricains sur 76 concernés dans le monde, avec 230 millions de personnes infectées sur 800 millions de personnes exposées et plus de 800000 décès annuels. L'objectif de cette étude était de tester l'efficacité d'une dose unique de Plaziquantel pour traiter les enfants d'âge scolaire de 11 villages maliens situés en zones endémiques de la schistosomiase urinaire. 
Après assentiment ou consentement, l'enregistrement des volontaires âgés de cinq ans ou plus a été fait. Après dépistage systématique de l'ensemble des volontaires pour savoir la prévalence de l'infection avant traitement, une dose unique de Praziquantel a été donnée aux sujets infectés. Un mois après la prise du médicament, un second dépistage a été fait chez les mêmes volontaires afin de mesurer l'effet du traitement. La technique de filtration de l'urine pour la détection de l'excrétion des œufs de schistosomiase a été utilisée comme méthode de diagnostic. Sur 549 volontaires testés à l'enregistrement (397 féminins et 152 masculins), 9,1\% (51/549) étaient infectés par $S$. haematobium. Un mois après le traitement à la dose unique de Praziquantel, un taux de réduction significatif de 3,5\% (P=0,03) a été constaté. Cette étude a montré que le Praziquantel reste toujours efficace pour le traitement de la schistosomiase urinaire. Le maintien de ce produit comme molécule distribuée pour le traitement de masse du programme national de lutte contre la schistosomiase est justifié.

Mots clés : Schistosomiase urinaire,charge ovulaire, traitement efficace, Praziquantel, Mali 


\title{
Efficacy of Praziquantel Single Dose Treatment of Urinary Schistosomiasis Among School Age Children from Endemics Area in Mali
}

\section{Alpha Seydou Yaro, Maitre de Conférences, PhD \\ Michel Emanuel Coulibaly, Master}

Faculté des Sciences et Techniques (FST), Université des Sciences, des Techniques et des Technologies de Bamako (USTTB), Mali ; International

Centre for Excellence in Research (ICER-Mali) Point G ; Faculté de

Médecine et d'Odontostomatologie (FMOS)

USTTB, Bamako, Mali

Yaya Coulibaly, Maitre-Assistant, PhD

International Centre for Excellence in Research (ICER-Mali) Point G Faculté de Médecine et d'Odontostomatologie (FMOS) ; USTTB, Bamako, Mali

Bernard Sodio, Professeur Titulaire, PhD

Faculté des Sciences et Techniques (FST), Université des Sciences, des

Techniques et des Technologies de Bamako (USTTB), Mali

Sekou F. Traoré, Professeur Titulaire, PhD

International Centre for Excellence in Research (ICER-Mali) Point G Faculté de Médecine et d'Odontostomatologie (FMOS) ; USTTB, Bamako, Mali

\begin{abstract}
In Mali, schistosomiasis is a public health problem as in all 42 African countries out of 76 affected worldwide, with 230 million people infected in 800 million people exposed and more than 800000 annual deaths. The objective of this study was to test the efficiency of a single dose of Plaziquantel to treat school-age children in 11 Malian villages located in endemic areas of urinary schistosomiasis. After consent, the enrolment of volunteers greater than 5 years old was done. After systematic screening of all volunteers for the prevalence of infection before treatment, a single dose of Praziquantel was given to infected individuals. One month after drug administration, a second screening was done among the same volunteers to measure the treatment effect. The technique of schistosomiasis eggs detection by urine filtration was used as a diagnostic method. From the screening including 549 volunteers (397 females and 152 males), 9.1\% (51/549) were positives to $S$. haematobiuminfection. One month after treatment with a single dose of Praziquantel, a significant rate reduction $(3.5 \%, \mathrm{P}=0.03)$ was observed. This study showed that Praziquantel is still effective for the treatment of urinary schistosomiasis. The choice of this drug by the national schistosomiasis control program for community mass treatment was justified.
\end{abstract}


Keywords: Urinary schistosomiasis, eggs load,effective treatment, Praziquantel, Mali

\section{Introduction}

La schistosomiase est une maladie parasitaire eau dépendante très répandue dans le monde, surtout en Afrique sub-saharienne (OMS, 2016). Jusqu'en 2011, la Bilharziose sévissait encore dans 76 pays situés en Afrique, en Amérique du sud, au Moyen Orient et au Sud du continent Asiatique (WHO, 2011). Deuxième endémie après le paludisme dans les régions tropicales et subtropicales (Engels et al., 2002 ; WHO, 2011), la schistosomiase ou bilharziose constitue un véritable problème de santé publique dans les pays endémiques dont 42 pays en Afrique (Traore et al., 2007 ; Clements et al., 2008 ; OMS, 2010). En effet certaines études ont montré que, près de 800 millions de personnes y sont exposées dans le monde (Aubry, 2016). Sur 230 millions de personnes infectées subissant le traitement annuel, 80 à 90\% vivent en Afrique avec 800000 décès par an (Aubry, 2016), dont 280000 cas de décès dus aux complications (Chippaux, 2000 ; Chitsulo et al, 2004 ; WHO, 2011).

$\mathrm{Au}$ Sud du Sahara, elle est la cause d'une hématurie et d'atteinte grave des parois chez des millions de personnes. La zone de prévalence se situe dans les régions tropicales et subtropicales des communautés vivant le long des cours d'eau. Beaucoup d'études ont confirmé que la répartition des schistosomiases était fortement liée à l'environnement physique. Par exemple, les aménagements hydro agricoles créent un contexte favorable au développement de cette maladie (Molyneux et al, 2005 ; Steinman et al, 2006 ; Garcia et al., 2013 ; Yapi et al, 2017). La coïnfection entre S. hoematobium, S. mansoniou bien entre S. hamatobiumet S. gueneesis(Moné et al, 2012) a été déjà documentée dans certains pays d'Afrique subsaharienne.

Au Mali, la schistosomiase a fait l'objet de beaucoup d'études sur l'épidémiologie (Tandina et al, 2016), la variabilité génétique, le degré de l'intensité de l'infection et enfin sur la morbidité ; surtout dans les zones endémiques. La Schistosomiase est endémique dans de nombreuses régions du pays (Traore et al, 2007 ; Clementset al, 2008). Mais les niveaux de transmission sont très variables d'une région à une autre et d'un village à un autre dans la même zone bioclimatique (Traore et al, 2007). Présentement, le Mali a un taux de prévalence global de $30 \%$ avec une différence entre les différentes zones endémiques. Les zones hyper endémiques qui sont les terres irriguées de l'Office du Niger à Ségou, le plateau Dogon avec des petits barrages, le bassin du fleuve Sénégal ont une prévalence supérieure à $50 \%$ (Steinman, 2006). Les zones soudaniennes et nord soudaniennes sont moins touchées avec une prévalence de 10\% (Traore et al, 2007). Actuellement dans 
la région de Ségou des études ont donné une prévalence de 79,8\% pour $S$. haematobiumet $13,2 \%$ pour $S$. mansoni(Stecher et al, 2017).

La chimiothérapie a été la stratégie de choix de l'OMS à travers les programmes nationaux de lutte contre la schistosomiase pour la prévention et la réduction de la morbidité. Ce programme existe au Mali depuis 1982 ; il est axé principalement sur le traitement de masse au Praziquantel dans la zone de l'Office du Niger et à Bandiagara. Actuellement le traitement de masse avec le Praziquantel au sein de la population à risque (les enfants d'âge préscolaire et scolaire, les pêcheurs, agriculteurs, les femmes etc.) demeure l'approche principale ayant le meilleur rapport coût /efficacité, la plus faisable et la plus durable. Malgré ces traitements annuels ou biannuels, il a été rapporté que la réinfection survient dans les zones endémiques en moins de 12 mois chez plusieurs enfants traités (N'Goran et al, 2001). C'est dans cette dynamique que cette étude a été entreprise afin de vérifier si la molécule utilisée pour les différents traitements demeure toujours efficace. Pour cela, nous avions mesuré l'effet d'une dose unique de traitement par le Praziquantel. Ces résultats pourront éclairer le programme national de lutte contre cette infection à revoir sa stratégie d'intervention en cas d'inefficacité constatée.

\section{Matériels et Méthodes}

\section{Présentation des sites d'études}

Cette étude s'est déroulée dans le district sanitaire de Kolokani situé à $105 \mathrm{~km}$ de Bamako (Figure 1, Source Unité GIS/RS du MRTC FMOS-USTTB Mali 2016). La zone a une superficie de $14380 \mathrm{~km}^{2}$ avec une population totale estimée à peu près à 287380 habitants. Un total de 11 villages des aires de santé de Tioribougou (13.382799 N ; -7.993163 W) et de Nonkon (13.222941 $\mathrm{N} ;-7.860675 \mathrm{~W}$ ) ont fait l'objet d'investigation (Figure 1). 

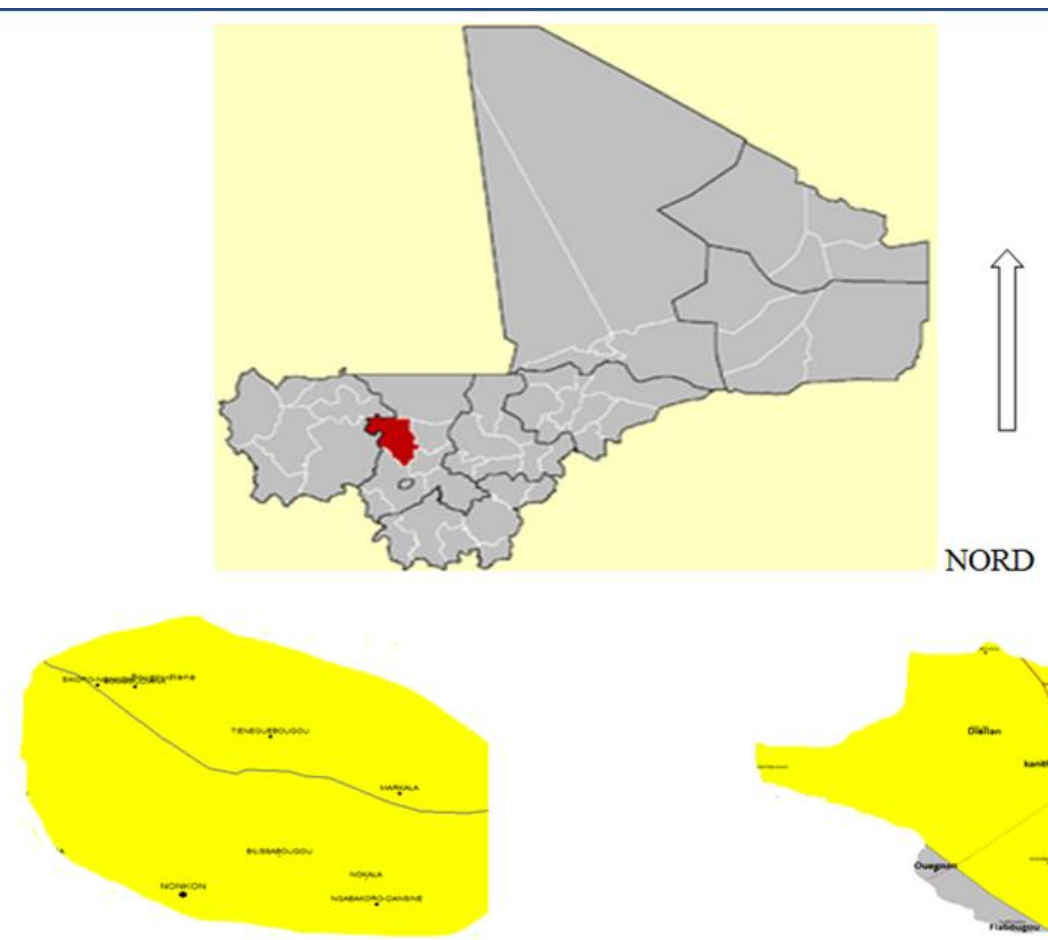

Aire de santé de Nonkon

- Village
- Routenationale(RN6
Lacpemanente

Figure 1 : Sites d'études, carte du Mali et les airs de sante de Nokon et de Tioribougouagrandis, Source Unité GIS/RS du MRTC FMOS-USTTB Mali

C'était une étude transversale à deux passages de prospection. Le premier passage de l'étude s'est déroulé en juillet 2015, phase avant distribution de masse de médicaments (DMM) et le deuxième passage en septembre 2015 (Phase après distribution de masse). Les critères d'inclusion étaient : être volontaire, être âgé de cinq ans ou plus, être résidant des aires de santé de Tioribougou et de Nonkon.

\section{Matériels utilisés}

Les matériels utilisés étaient entre autres :

- Pot d'urine : Pour la collecte des échantillons d'urine

- Seringue $10 \mathrm{cc}$ : Pour prendre les $10 \mathrm{cc}$ d'urine et les filtrer

- Membrane nucléo pore : Pour filtrer et retenir les œufs de $S$. haematobium 
- Tables : Pour servir de surface de travail pour les techniciens

- -Marqueurs: Pour marquer les numéros d'identification des volontaires sur les matériels de travail comme les pots, les lames.

- Pinces : Pour manipuler les membranes nucléo-pores

- $\quad$-Ninhydrine 3 ou 5\% : Pour colorer les œufs de S. haematobium.

- -Microscope binoculaire : Pour observer les œufs sur les membranes nucléopores déposées sur les lames porte-objet.

- Source d'électricité : Pour alimenter les microscopes.

- -Plateau : Pour contenir les lames préparées pour la lecture.

- -Bassine : Pour laver les pots d'urines.

- -Porte filtre: Pour porter les membranes nucléopores pendant la filtration.

\section{Organisation des équipes de travail}

Le travail était reparti entre deux postes :

- le poste d'enregistrement des volontaires où est attribué un numéro unique d'identification pour chaque volontaire éligible. Le volontaire est photographié avec son numéro d'identification lisiblement exhibé. Ensuite il reçoit un bocal (pot en plastique) pour le recueil de son urine.

- le poste de diagnostic où l'urine est réceptionnée et examinée par des agents qualifiés.

Les urines étaient prélevées de préférence au milieu de la miction après quelques exercices physiques simples entre 10 heures et 14 heures. Pour la détection des œufs de $S$. haematobium, $10 \mathrm{ml}$ d'urine par volontaire ont été filtrés immédiatement après la collecte sur une membrane nucléopore; ensuite ces filtres qui avaient retenus les œufs de S. haematobium étaient colorés à la Ninhydrine puis séchés et conservés pour être lus au microscope.

L'ensemble du travail est fait dans un centre privé localisé dans le village de Tienéguébougou. Ce centre est construit et équipé par les partenaires américains du Centre International pour l'Excellence en Recherche au Mali (ICER-Mali).

\section{Mode Opératoire}

- Inscrire le numéro du volontaire sur la membrane nucléo-pore,

- Placer la membrane dans un porte-filtre, bien adapté de manière à éviter que l'urine ne s'écoule au moment de la filtration,

- prélever $10 \mathrm{ml}$ d'urine à l'aide d'une seringue adaptable au porte-filtre,

- pousser le piston pour chasser l'urine à travers le filtre tout en maintenant la seringue verticale,

- enlever la seringue du porte-filtre, tirer une nouvelle fois le piston, puis chasser le reste des urines du filtre, 
- ouvrir le porte-filtre et déposer à l'aide d'une pince le filtre sur une lame porte-objet.

- -déposer une goutte deNinhydrine sur le filtre,

- - laisser sécher le filtre,

- monter le filtre sous microscope puis chercher et compter le nombre d'œufs de S. haematobium, pour déterminer la charge ovulaire.

Selon le barème de l'OMS (OMS, 2013), le nombre d'œufs par 10ml d'urine et l'intensité de l'infection sont définis comme suit :

*forts excréteurs : $\geq 50$ œufs $/ 10 \mathrm{ml} ;$ *faibles excréteurs : $<50$ œufs $/ 10 \mathrm{ml}$

Ainsi les forts excréteurs sont les volontaires ayant plus de 50 œufs de $S$. haematobium dans un volume de $10 \mathrm{ml}$ d'urine et les faibles excréteurs sont ceux ayant moins de 50 œufs dans le même volume d'urine (OMS, 2013).

\section{Saisie et analyse des données}

Les données ont été saisies dans le logiciel Excel, puis analysées par Epi info 7 et SPSS 16.0, les données sont présentées sous forme de tableaux ou de figures. Les tests de $\mathrm{Chi}^{2}$ et de Fisher exact ont été utilisés pour comparer les proportions. Pour les tests statistiques, les valeurs de $\mathrm{P}<0,05$ ont été considérées comme statistiquement significatives.

\section{Considérations éthiques}

Avant le démarrage de l'étude, des rencontres d'explication et de sensibilisation du protocole ont été tenues. D'abord nous avions eu le consentement global de la communauté. Cela a été confirmé par un consentement individuel pour les volontaires majeurs et l'assentiment des parents pour les mineurs de 5 à 17 ans. Les volontaires étaient libres de se retirer de l'étude à tout moment et le traitement était gratuit pour tous.

\section{Résultats}

\section{Données démographiques}

Les investigations ont été faites dans 11 villages. Sur un total de 549 sujets enregistrés, 397 individus étaient de sexe féminin et 152 de sexe masculin (Tableau I). Le village de Tiénéguébougou a enregistré le plus grand nombre de sujets (91). Le tableau (Tableau I) indique aussi la répartition des sujets volontaires selon le sexe et la localité d'appartenance. Les sujets féminins étaient plus représentés 72,3\% (397/549) comparés aux sujets masculins (152/549) soit 27,7\%. Cette supériorité numérique des sujets féminins par rapport aux masculins est constatée dans tous les villages. 
Tableau I : Répartition par sexe et par village des sujets volontaires ayant rempli les critères d'inclusion de l'étude.

\begin{tabular}{lccc}
\hline Village & Féminin & Masculin & Total par village \\
\hline Tiénéguébougou & 60 & 31 & 91 \\
Ouégnan & 47 & 29 & 76 \\
Flabougou & 45 & 8 & 53 \\
Niara & 45 & 8 & 53 \\
Sikoro & 42 & 11 & 53 \\
Kanekébougou & 31 & 18 & 49 \\
Soninkoro & 28 & 12 & 40 \\
Dialan & 34 & 3 & 37 \\
Bougoudiana & 24 & 13 & 37 \\
Doribougou & 23 & 10 & 33 \\
Galamado & 18 & 9 & 27 \\
Total par sexe & $\mathbf{3 9 7}$ & $\mathbf{1 5 2}$ & $\mathbf{5 4 9}$ \\
\hline
\end{tabular}

\section{Données Parasitologiques et cliniques}

*Prévalence de la schistosomiase urinaire dans la population d'étude avant le traitement

Avant le traitement de masse avec le Praziquantel, la prévalence moyenne dans les 11 villages de l'étude était de 9,3\%, les prévalences les plus élevées ont été enregistrées à Flabougou, Kanekébougou, Tiénéguébougou et Ouegnan avec respectivement 24,5\%, 16,3\%, 14,3\% et 13,2\% (Figure 2).

Après traitement avec une dose unique de Praziquantel, la prévalence moyenne a chuté de 9,3 à $5,8 \%$ soit une réduction de 3,5 au sein de la population d'étude globale. Cette diminution a pratiquement été constatéeà l'échelle de chaque village, mais beaucoup plus accentuée dans les villages de Flabougou, Tiénéguébougou, Kanekébougou et Ouegnan, où les prévalences constatées après traitement étaient respectivement $17 \%, 12,1 \%, 8,2 \%$ à et 3,9\% (Figure 2). 


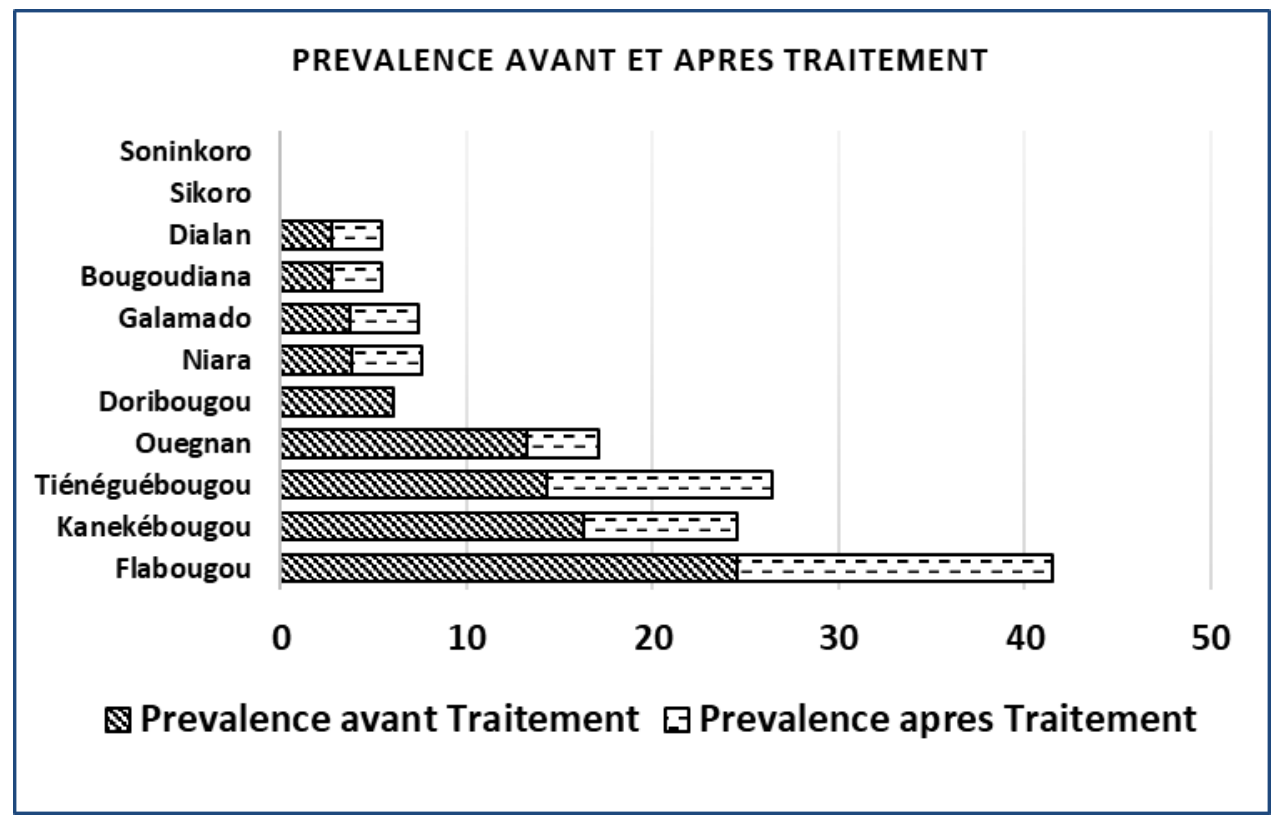

Figure 2 : Prévalence de la schistosomiase urinaire avant et après traitement de masse avec dose unique de Praziquantel dans les différents villages d'étude.

Avant le traitement, l'ensemble des forts excréteurs venaient de cinq villages alors qu'après traitement, les forts excréteurs n'ont été rencontrés que dans trois villages : Kanekébougou, Flabougou et Tiénéguébougou avec des prévalences respectives de $4,1 \% ; 3,8 \%$ et 3,3\% (Figure 3-A).

Par contre, à l'exception de trois villages avant traitement et 3 trois villages après traitement, les faibles excréteurs ont été rencontrés dans tous les autres villages (Figure 3-B), mais les plus fortes prévalences ont été rencontrées dans les villages de Flabougou, Tiénéguébougou et Kanekébougou avec une prévalence respective de $17 \%, 11 \%$ et $8,2 \%$ avant traitement. Et après traitement, ces prévalences ont chutées à $3,8 \% ; 13,2 \%$; $8,8 \%$ et $4,1 \%$ respectivement pour Flabougou, Tiénéguébougou et Kanekébougou (Figure 3-B). 


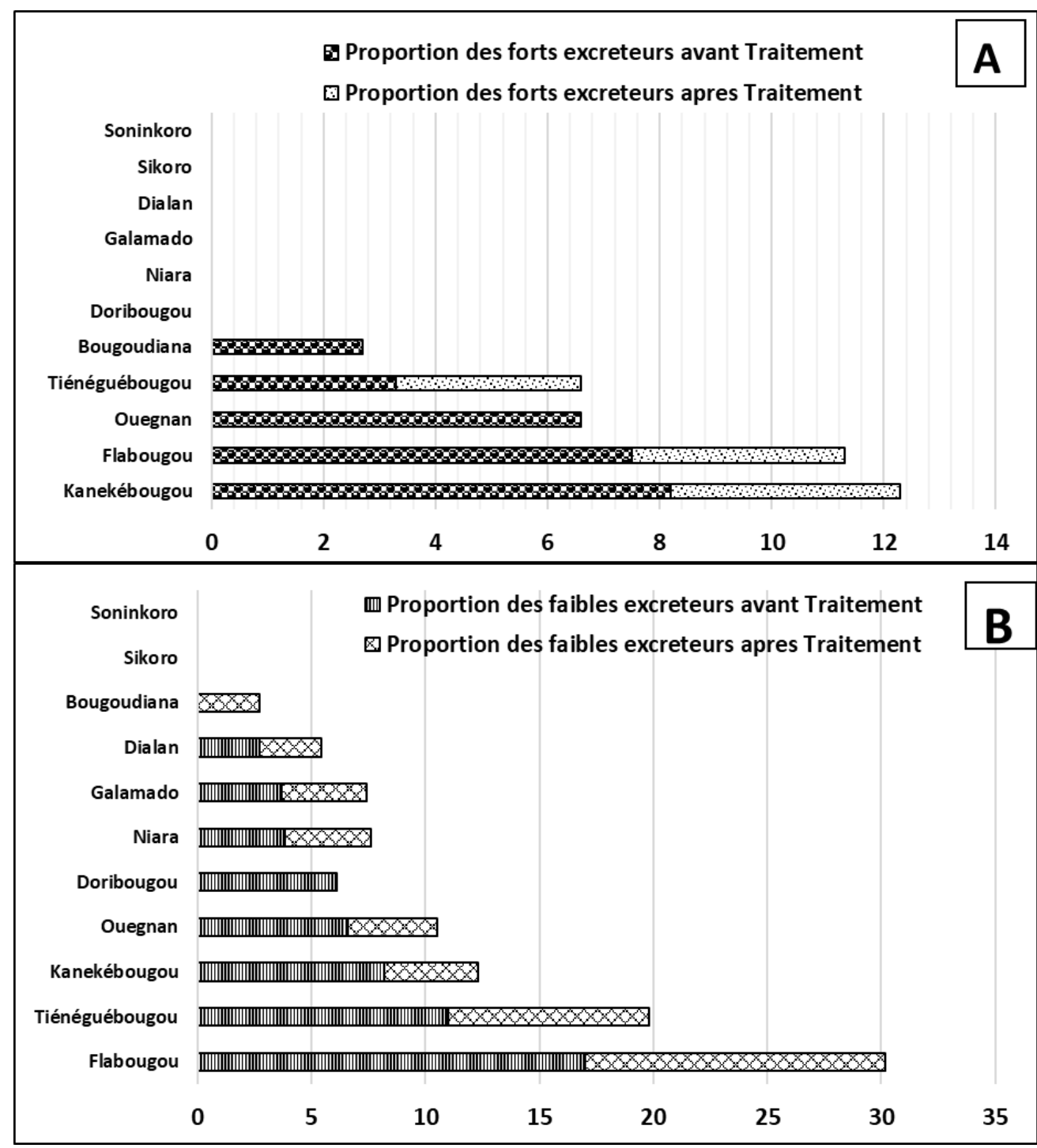

Figure 3 : Intensité de l'infection due à S. haematobium avant et après traitement de masse des volontaires avec une dose unique de Praziquantel à l'échelle de chaque village chez les forts excréteurs (A), et chez les faibles excréteurs (B).

Avant Traitement, sur 53 sujets infectés par S. haematobium dans la population d'études globale, 34 individus étaient des faibles excréteurs et 17 individus étaient des forts excréteurs (Figure 4-A et $\boldsymbol{B})$. Un mois après traitement de masse avec la dose unique de Praziquanteldans les différents villages d'étude, le nombre de faibles excréteurs a chuté de 34 à 25 avec une réduction significative de 6,19\% (34/549) à 4,6\% (25/549), (p=0.02) (fig. 11). (Figure 4-B). Par contre, le nombre de forts excréteurs a chuté de 17 à 7 
individus avec une réduction statistiquement non significative $(\mathrm{p}=0,06)$ (Figure4-A).
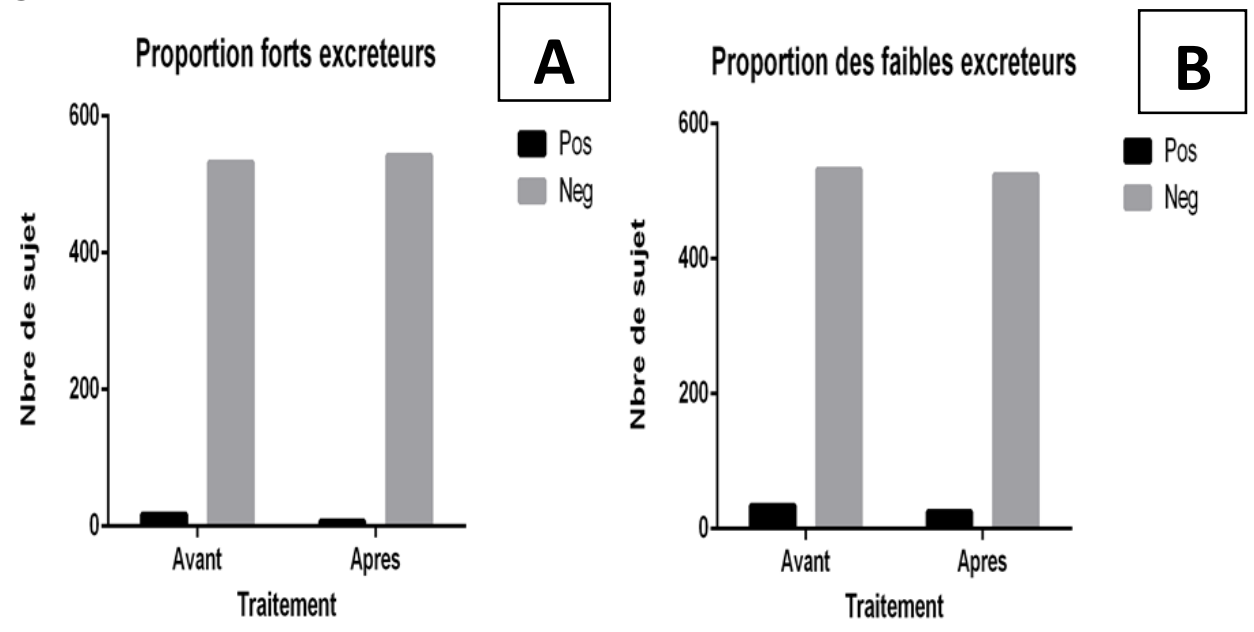

Figure 4 : Mesure de l'intensité moyenne de l'infection due à $\mathrm{S}$. haematobium avant et après traitement de masse des volontaires avec une dose unique de Praziquantel et à

l'échelle globale au niveau des forts excréteurs (A) et des faibles excréteurs (B)

\section{Discussions}

Beaucoup d'organismes animaux (Karamoko et al, 2016 ; Youssef et al, 2016) aussi bien que humains (Aziz et al, 2016 ; Hummar et al, 2017) sont colonisés par les parasites. La présente étude fait allusion à l'effet d'un médicament sur les parasites de la bilharziose au sein d'une population vivant en zone endémique. Le Praziquantela été utilisé comme médicament de traitement de masse.

Sur un total de 549 volontaires dont 397 féminins et 152 masculins, le taux d'infection moyen pour la bilharziose à S. haematobium était de 9,3\% (51/549) dans la population d'étude globale. La forte participation des sujets de sexe féminin par rapport aux masculins avait déjà été signalée par Ly et al, 2019.

Suite à un traitement de masse à dose unique de Praziquantel, avec un taux de couverture de 66,67\% (366/549), nous avions mesuré l'effet de l'intervention un mois après. Une baisse statistiquement significative du taux d'infection de 9,3\% (51/549) à 5,8\% (32/549) a été constatée à l'issue d'un mois $(\mathrm{P}=0,039)$. Ces résultats sont similaires à ceux de Sacko et al, 2005 qui ont trouvé des prévalences respectives de $11,3 \% ; 24 \% ; 8,8 \%$ et $11,6 \%$ respectivement à Kermis, Mouline, Bafoulabe et Koundia. Ces résultats corroborent également ceux de Bagagan et al, 2016 qui ont aussi trouvé une prévalence de $8,1 \%$ en 2016 au Burkina Faso. Le rapport annuel du programme national de lutte contre la schistosomiase et les géohelminthiases en 2015 a rapporté des prévalences similaires de 10\% à Dialakoroba dans la 
région de Koulikoro au Mali. Par contre, Ly et al, 2019 ont trouvé une prévalence globale plus élevée de $56,7 \%$ d'infection par $S$. haematobium dans trois villages situés le long du fleuve Niger au Mali. Mais une prévalence plus élevée a été rencontrée au Mali par d'autres auteurs comme Kouriba qui avait abouti à une infection de $100 \%$ chez les enfants de 4 à 7 ans à Bandiagara au cours d'une étude sur l'immunité anti bilharzienne en 2004; Traoré (2006) avait trouvé $73,90 \%$ d'infection de schistosomes chez des enfants de Dialakoroba au cours d'une étude de coinfection Schistosomiase-Paludisme chez des sujets de 4 à 14 ans. La différence entre les taux de prévalence de la présente étude comparée à celles de Kouriba et al, 2004, Traoré et al, 2006 et Ly et al, 2019 est que les différentes études ont été faites dans des zones à risques inégaux; car les autres ont travaillé dans les zones ayant les plus forts taux de prévalence au Mali à cause des facteurs d'exposition dont ils disposent. Avec une prévalence initiale globale de 9,3\% avant traitement, une réduction significative due à l'effet du traitement de masse a abaissé la prévalence moyenne globale à 5,8\%. Au-delà des données globales, cette tendance a été visible même à l'échelle des villages, aussi bien chez les forts excréteurs que chez les faibles excréteurs. Au Niger,Garba et al en 2000 avaient eu aussi une réduction plus forte, allant de $98 \%$ à $32 \%$ avec une population d'étude composée de 318 élèves âgés de 6 à 16 ans. Cela pourrait être dû au fait que dans certaines zones la sensibilisation est plus facile et la réinfection est lente. L'intensité de l'infection a été réduite après une seule séance de traitement avec le Praziquantel. La moyenne géométrique calculée chez les forts excréteurs avec $10 \mathrm{ml}$ d'urine avant le traitement au Praziquantel était de 7,93 œufs et après le traitement cette moyenne a été réduite à 5,35 œufs. Le traitement de masse avec le Praziquantel a considérablement diminué allant de $100 \%$ à $41,2 \%$ (17/17 à 7/17) chez les forts excréteurs. Ces résultats sont différents de ceux de Garba et al, 2000 qui ont eu, en six semaines après le traitement de masse avec le Praziquantel, une proportion des forts excréteurs réduitede $43,1 \%$ à $0 \%$ au Niger. Une identification par les moyens de biologie moléculaire (PCR) pourrait donner un résultat plus important comme reporté par Nanoukonet al (2017)

Par rapport aux sujets faibles excréteurs, une réduction de 6,19\% (34/549) avant traitement à 4,6\% (24/549) après traitement a été obtenue. Ces résultats sont contraires à ceux obtenus par Fatoumata (2011) qui avait plutôt obtenu une augmentation allant de $33,8 \%$ à $43,5 \%$ après traitement dans la région de Ségou chez les faibles excréteurs. Cela peut être dû au fait qu'à Ségou, le site d'étude était localisé au bord du fleuve Niger où les facteurs de réinfections rapides sont nombreux.Selon le barème de classification de l'OMS (2013) notre zone d'étude est classée parmi les communautés à risque modéré.Parmi les causes connues de l'infection schistosomienne, la non disponibilité d'eau potable est un facteur de risque important, car à défaut 
d'eau potables c'est les eaux de rivières ou de mares insalubres qui sont utilisées par la communauté.

\section{Conclusion}

La prévalence de la schistosomiase urinaire a significativement diminué de 9,3\% à 5,8\% après une seule dose de traitement de masse avec le Praziquantel. Les analyses statistiques ont montré une réduction significative du taux de prévalence moyenne globale entre la situation avant traitement et la situation post traitement. Une réduction de taux de prévalence a été aussi constatée chez les faibles excréteurs et les forts excréteurs aussi bien à l'échelle des villages qu'à l'échelle de la population globale de l'étude.

Cette étude a montré que le Praziquantel reste encore efficace pour le traitement des populations soufrant de la bilharziose urinaire dans les zones endémiques au Mali.

\section{References:}

1. Aldhoun JA, Littlewood DTJ. Orientobilharzia Dutt \& Srivastava, (1955) Trematoda: Schistosomatidae, a junior synonym of Schistosoma Weinland, 1858., Systematic Parasitology. Vol. 82 ; 2012. p. 81-8.

2. Arene F, Ukpeibo ET, Nwanze EA. (1989). Studies on schistosomiasis in the Niger Delta: Schistosoma intercalatum in the urban city of Port Harcourt, Nigeria. Public Health.;103(4):295-301.

3. Aziz, E. A., Alaoui, Z., Khadija, E. K., Driss, B., Yassine, A., Mohamed, H. (2016). Profil Epidemiologique de la Leishmaniose cutanee dans la région du Gharb- Maroc de 2006 à 2014.European Scientific Journal January 2016 edition vol.12, No.3 ISSN: 1857 7881 (Print) e - ISSN 1857- 7431. doi: 10.19044/esj.2016.v12n3p243.

4. Becquet R, Saout J. (1969). Intestinal bilharziasis caused by Schistosoma intercalatum in Upper Volta . Bull Soc Pathol Exot Filiales. Jan-Feb;62(1):146-51.

5. Betterton C, Fryer SE, Wright CA. (1983). Bulinus senegalensis (Mollusca Planorbidae) in northern Nigeria_Ann Trop Med Parasitol. 1983 Apr;77(2):143-9. DOI:10.1080/00034983.1983.11811689

6. Combes C. (1990). Where do human schistosomes come from? An evolutionary approach. Trends Ecol Evol.;5(10):334-7.

7. Corachan M, Escosa R, Mas J, Ruiz L, Campo E. (2016) Clinical presentation of schistosoma interculatum infestation. Lancet. 16 mai 1987 ;329(8542):1139-40. Disponible sur:

http://www.thelancet.com/article/S0140673687916904/

8. Dabo A, Diop S, Doumbo O.(2017). Distribution des mollusques hôtes intermédiaires des schistosomiases humaines à l'office du Niger 
(Mali). II: Rôle des différents habitats dans la transmission. Bull la Société Pathol Exot];87(3):164-9. Disponible sur: http://cat.inist.fr/?aModele=afficheN\&cpsidt $=3381373$

9. Dabo A, Sissoko M, Audibert M, Diakité M, Diarra A, Diallo M, et al. (2005). Impact de la chimiothérapie de masse au PZQ sur l'infection due à Schistosoma haematobium et Schistosoma mansoni à l' Office du Niger, Mali . Impact of mass chemotherapy with praziquantel on Schistosoma haematobium and Schistosoma mansoni inf.;29-33

10. Dabo A, Traore HA, Diakite M, Kouriba B, Camara F, Coulibaly CO, et al. (1995) [Echographic morbidity due to Schistosoma haematobium in a peripheral district of Bamako in Mali, Missabougou]. Bull Soc Pathol Exot ;88(1):11-4. Disponible sur:

http://www.ncbi.nlm.nih.gov/pubmed/7787444*

11. Doumenge J.P. Atlas de la répartition mondiale des schistosomiases/Atlas of the Global Distribution of Schistosomiasis. Presses universitaires de Bordeaux. Livre broché. pages 402 p. ISBN10 2-86781-060-4 ; ISBN-13 9782867810602

12. Gryseels B, Nkulikyinka L. (1990). The morbidity of schistosomiasis mansoni in the highland focus of Lake Cohoha, Burundi. Trans R Soc Trop Med Hyg.;84(4):542-7.

13. Guyatt HL, Brooker S, Donnelly C A. (1999). Can prevalence of infection in school-aged children be used as an index for assessing community prevalence? Parasitology;118 ( Pt 3:257-68.: http://www.ncbi.nlm.nih.gov/pubmed/10205801

14. Haas W, Haberl B, Schmalfuss G, Khayyal MT (1994). Schistosoma haematobium cercarial host-finding and host-recognition differs from that of S. mansoni. J Parasitol;80(3):345-53. http://www.ncbi.nlm.nih.gov/pubmed/8195934

15. Haberl B, Kalbe M, Fuchs H, Ströbel M, Schmalfuss G, Haas W. (1995).Schistosoma mansoni and S. haematobium: Miracidial hostfinding behaviour is stimulated by macromolecules. Int $\mathbf{J}$ Parasitol.;25(5):551-60.

16. Hammour, H., El Kharrim, K., Belghyti, D., (2017). Prospective Study of Intestinal Parasites in Children Hospitalized in the Pediatric Department at the Hospital in El Eldrissi (Kenitra City - Morocco). European Scientific Journal, edition Vol.13, No.18 ISSN: 1857 - 7881 (Print) e - ISSN 1857- 7431. doi: 10.19044/esj.2017.v13n18p520.

17. He YX, Chen L, Ramaswamy K. (2002). Schistosoma mansoni, S. haematobium, and $S$. japonicum: Early events associated with penetration and migration of schistosomula through human skin. Exp Parasitol.;102(2):99-108. 
18. Jauréguiberry S., Paris L., Caumes E. (2010) Acute schistosomiasis, a diagnostic and therapeutic challenge. Clinical Microbiology and Infection. Volume 16, Issue 3, Pages 225-231. https://doi.org/10.1111/j.1469-0691.2009.03131.x.

19. Kalbe M, Haberl B, Hertel J, Haas W. (2004). Heredity of specific host-finding behaviour in Schistosoma mansoni miracidia. Parasitology ;128:635-43. Disponible sur: Go to ISI://WOS:000221987700008

20. Karamoko, M., Amani, N. S., Touré, A., Komoin, O, C., Otchoumou, A., tcho Fantodji, A., Kouassi, K, P., (2016). Prevalence Of African Giant Snails For Parasites In A South-East Region Of Côte d'Ivoire. European Scientific Journal July edition vol.12, No.21: ISSN: 1857 7881 (Print) e - ISSN 1857- 7431. doi:10.19044/esj.2016.v12n21p186

21. Karanja DMS, Colley DG, Nahlen BL, Ouma JH, Secor WE. (1997). Studies on schistosomiasis in western Kenya: I. Evidence for immunefacilitated excretion of schistosome eggs from patients with Schistosoma mansoni and human immunodeficiency virus coinfections. Am J Trop Med Hyg.;56(5):515-21.

22. Kjetland EF, Ndhlovu PD, Kurewa EN, Midzi N, Gomo E, Mduluza $\mathrm{T}$, et al. Prevention of gynecologic contact bleeding and genital sandy patches by childhood anti-schistosomal treatment. Am J Trop Med Hyg. 2008;79(1):79-83.

23. Mc Kerrow JH. (1997). Cytokine induction and exploitation in schistosome infections. Parasitology.;115 Suppl:S107-12. : http://www.ncbi.nlm.nih.gov/pubmed/9571696

24. Moné H, Minguez S, Ibikounlé M, Allienne J-F, Massougbodji A, Mouahid G. (2012). Natural Interactions between S. haematobium and S. guineensis in the Republic of Benin. Sci World J;2012:1-8.: http://www.hindawi.com/journals/tswj/2012/793420/

25. Nanoukon, C, N, M., Dassou, E, G., Dousso, M., Sogbo, F., Sina H., Prevost G., Lamine Moussa, L, B (2017) Staphylococcus Epidermidis Producteur d'entérotoxine $\mathrm{C}$ impliqué dans une septicémie à foyer urinaire. European Scientific Journal August 2017 edition Vol.13, No.24 ISSN: 1857 - 7881 (Print) e - ISSN 1857- 7431 doi:10.19044/esj.2017.v13n24p278 http://dx.doi.org/10.19044/esj.2017.v13n24p278

26. N'Goran E, Brémond P, Sellin E, Sellin B, Théron A. (1997). Intraspecific diversity of Schistosoma haematobium in West Africa: Chronobiology of cercarial emergence. Acta Trop.;66(1):35-44.

27. OMS. Impact de la schistosomiase sur la santé publique: morbidité et mortalité *. Schistosomatidae Poche, 1907. 1907;55312. 1994;72(1):5-11. 
28. OMS (2013). Schistosomiase: Rapport de situation 2001-2011 et plan stratégique 2012-2020. 2013;80.

29. Poche, Aspidogasteridae (1907) Schistosomatidae. Kingdom Animalia. 1907;55312.

30. Poda J-N, Sellin B, Sawadogo L, Sanogo S. (1994). Distribution spatiale des mollusques hôtes intermédiaires potentiels des schistosomes et de leurs biotopes au Burkina Faso. Occge Info.;(101):12-9.

31. Salter JP, Lim KC, Hansell E, Hsieh I, McKerrow JH. (2000) Schistosome invasion of human skin and degradation of dermal elastin are mediated by a single serine protease. J Biol Chem. 2000;275(49):38667-73.

32. Selling B et Boudin C. (1981). Les schistosomes en Afrique de l' Ouest. ANNALE de Parasitologie de I'O.R.S.T.O.M.

33. Stelma FF, Talla I, Polman K, Niang M, Sturrock RF, Deelder AM, et al. (1993). Epidemiology of Schistosoma mansoni infection in a recently exposed community in Northern Senegal. Am J Trop Med Hyg.;49(6):701-6.

34. Surugue, B. (1993). La lutte contre les maladies tropicales: la schistosomiase. OMS.

35. Tandina F, Doumbo SN, Kone AK, Guindo D, Goita S, Sissoko M, Konate S,. Dabo A, Doumbo OK (2016). Épidémiologie des schistosomoses dans le village périurbain de Sotuba, dix années après la mise à échelle du traitement de masse au Mali. Medecine et Sante Tropicales, 26: 51-56. DOI:10.1684/mst.2015.0515.

36. Traoré M . (1990) - Répartition des schistosomiases au Mali - Act. Conf. Internat. Schisto. Niger CERMES/OCCGE: 103-135. 1990;1990.

37. Traoré M, Landoure A, Diarra A, Kante B, Sacko M, Coulibaly G, et al. (2007). Geographic distribution and epidemiology of urinary schistosomiasis in Mali: implications for a control program. Mali Med ;22(3):22-8.: http://www.ncbi.nlm.nih.gov/pubmed/19434989

38. Traoré M, Maude GH, Bradley DJ. (1998). Schistosomiasis haematobia in Mali: prevalence rate in school-age children as index of endemicity in the community. Trop Med Int Health.;3(3):214-21.

39. Vester U, Kardorff R, Traoré M, Traoré HA, Fongoro S, Juchem C, et al. (1997). Urinary tract morbidity due to Schistosoma haematobium infection in Mali. Kidney Int.;52(2):478-81. Disponible sur: http://www.ncbi.nlm.nih.gov/pubmed/9264005

40. Vray B. (2002). Les apports de la biologie moléculaire à la paléoparasitologie. Vesalius.;3(1):45-52. 
41. Wang L, Li YL, Fishelson Z, Kusel JR, Ruppel A. (2005). Schistosoma japonicum migration through mouse skin compared histologically and immunologically with $S$. mansoni. Parasitol Res.;95(3):218-23.

42. Whitfield PJ, Bartlett A, Khammo N, Brain APR, Brown MB, Marriott C, et al. (2003). Delayed tail loss during the invasion of human skin by schistosome cercariae. Parasitology ;126(Pt 2):135-40: http://www.ncbi.nlm.nih.gov/pubmed/12636351

43. Wolmarans CT, de Kock KN, Strauss HD, Bornman M. (2002) Daily emergence of Schistosoma mansoni and S. haematobium cercariae from naturally infected snails under field conditions. J Helminthol.;76(3):273-7. http://www.ncbi.nlm.nih.gov/pubmed/12363382

44. Youssef, E., Taoufik, H., Driss, L., Brahim, C., Hajar, D., Nizar, S., Sanaa, Y., Khadija, E. K., Driss, B. (2016). Distribution spatiale de Rhadinorhynchus cadenti parasites intestinal de poisson Trachinotus ovatus de la Cote de Mehdia (Maroc).European Scientific Journal April 2015 edition vol.11, No.12 ISSN: 1857 - 7881 (Print) e - ISSN 1857- 7431.

45. Zussman RA, Bauman PM, Petruska JC. The role of ingested hemoglobin in the nutrition of Schistosoma mansoni. J Parasitol. 1970;56(1):75-9. 